\title{
Image Enhancement Technique Applied to Low-field MR Brain Images
}

\author{
Dr. Samir Kumar Bandyopadhyay \\ Professor \\ University of Calcutta \\ India
}

\begin{abstract}
Image processing techniques are used to extract meaningful information from medical images. A major concern in denoising low-field MR brain images is the poor quality images secondary to a worsening signal-to-noise ratio (SNR) compared with the high-field MRI scanners. Low-field Magnetic Resonance Imaging (MRI) is vital in sensitive surgeries to allow real-time imaging in the operation theatre. Since low-field MRI uses low strength electromagnetic fields, noisy low resolution images are produced. In contrast, highfield MRI machines (approximately 7T) are able to produce clear detailed images with almost no noise at all. Considering the above, it is required to enhance the low-field images, so that the same conventional and high-field MRI processing techniques and applications could be applied to pre-processed low-field MRI images. In this paper, pre-processing steps are applied to low-field MR brain images for improving quality of the image.
\end{abstract}

Key-Words: - Magnetic resonance imaging (MRI), Image analysis, and Image Enhancement

\section{INTRODUCTION}

Brain tumor is one of the major causes for the increase in Mortality among children and adults. A tumor is a mass of tissue that grows out of control of the normal forces that regulate growth [1]. Most Research in developed countries show that the number of people who develop brain tumors and die from them has increased rapidly over past three decades. The overall annual incidence of primary brain tumors in the U.S is 11 to 12 per 100,000 people for primary malignant brain tumors, that rate is 6 to 7 per $1,00,000$. In the UK, over 4,200 people are diagnosed with a brain tumor every year (2007 estimates In India, totally 80,271 people are affected by various types of tumor (2007 estimates). National Brain Tumor Foundation (NBTF) reported highest rate of primary malignant brain tumor occurred in Northern Europe, United States and Israel. Lowest rate arises in India and Philippines. The meninges are affected by a type of tumor called meningioma [2]. The affected rate given by World Health Organization (WHO) in the year 2007 is $7.06 \%$. Now rate is much higher than the given rate.
Open low-field-strength MRI units are popular imaging tools because claustrophobic patients tolerate them better than the more confining high-field-strength units. Some obese patients fit only into open units. Most low-field-strength units also cost less to purchase and maintain than high-field-strength units. However, often the low-field-strength units do not have fat suppression, and imaging takes considerably longer. The additional imaging time increases the risk of patient motion and resultant motion artifacts.

The strength of the magnetic field is measured in gauss or tesla units $(10,000 \mathrm{G}=1 \mathrm{~T})$. In general, higher field strength improves the signal-to-noise ratio (SNR). SNR, contrast, and resolution increase almost linearly with field strength, at least up to $1.5 \mathrm{~T}$. The increases allow faster scanning and lower the incidence of motion artifacts [3-4].

Image noise can affect visualization of low-contrast lesions. The ratio of signal difference (or contrast) to noise indicates how well a lesion can be detected. Faster scanning times and thinner slices tend to reduce noise. Image noise adversely affects the reviewer's ability to detect low-contrast lesions. Noise is reduced relative to signal by increasing voxel volume. Voxel volume can be increased by increasing the field of view or increasing the slice thickness. However, higher voxel volume may cause volume-averaging signal abnormalities that make lesions more difficult to detect

Figure 1 shows the differences in conventional and low-field MRI images. Both images were taken from the same patient, for the same slice of the brain. However, the quality is noticeably worse in the low field image. Not only is the noise level increased, but the orientation and coverage of the brain is affected as well due to the small aperture of the low-field machine. The lower part of the brain is no longer visible and an artifact is present at the top left (although not very visible in this image, it can hinder automatic processing). Low-field MRI usually has a black or gray background with an elliptic artifact. The background should be removed as it is not part of the brain. This technique has been used in [5] as one of the pre-processing steps in brain region extraction. In [6], it was expected that a distinct peak in the histogram exist for determining the threshold value for the entire image, such that the image could be properly enhanced. 


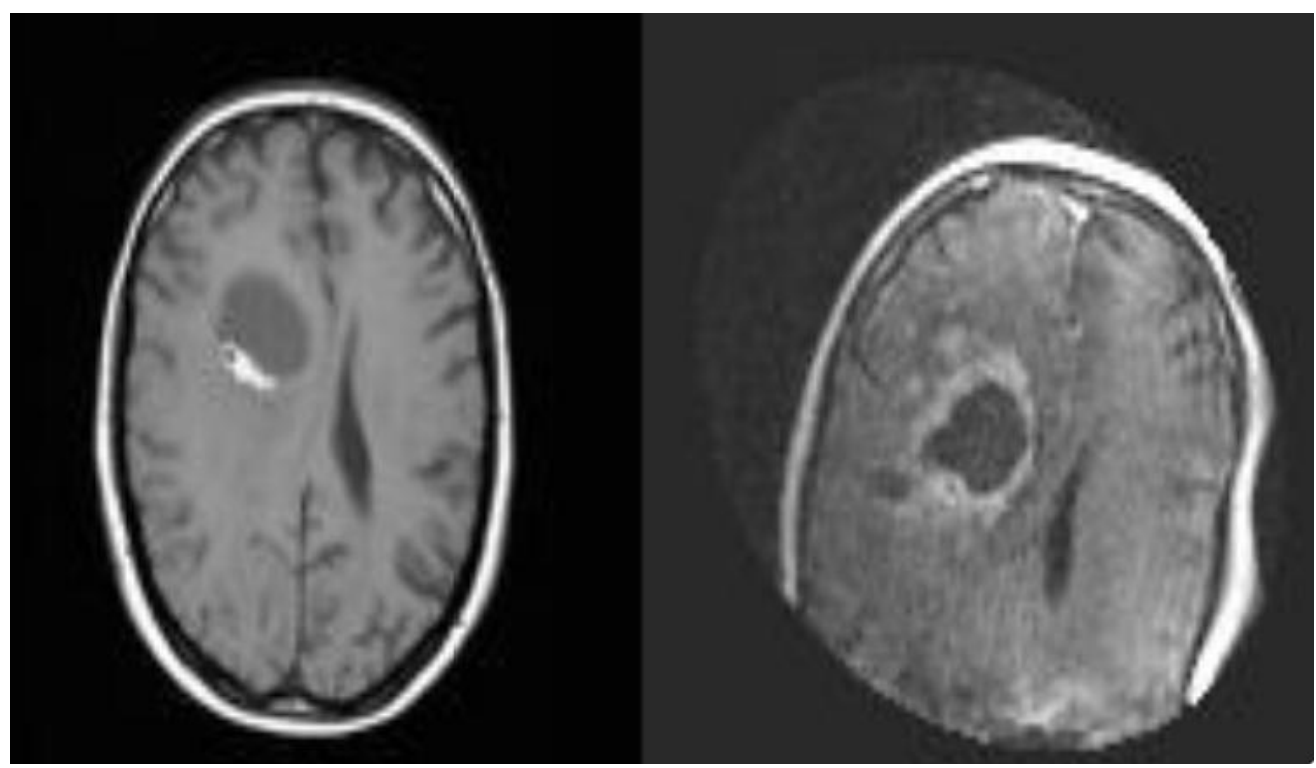

Figure 1 Conventional MRI (left), low-field MRI (right)

As low-field MRI equipment is still quite rare, a database of only 114 patients was available in this work. Each $128 \times 128$ pixels image was analysed. The parts of interest in a typical low-field MRI brain image are pre-processed to exclude the artifact and background.

\section{BACK GROUND OF PRE- PROCESSING IMAGE}

Images usually contain one or more type of noise and artifact. In medical images, because of diagnostic and therapeutic applications, this issue is critical. Specially in MR images, inhomogeneous magnetic fields, Patient motions duration imaging times, thermal noise and exist of any metal things in imaging environment, are some reasons that can create noises and artifact, though in most of times, are not very important because of human studies on images, but these are one of the main causes for computational errors in automatic or semiautomatic image analysing methods and so it is needed to be removed by pre-processing procedures before any analysing.
Here, pre-processing is equal to remove seeds from images and increase contrast between normal and abnormal brain tissues. The procedure have been used here are Histogram equalization, using Median filter, using Un sharp mask, thresholding and using from Mean filter respectively for each image. In this step, two dimensional discrete Fourier transform is computed for images. To reduce the noise a 3 by 3 pixel mean filter was implemented. This filtered averaged 9 points thus reducing the noise by 3 . Because a single pass of this filter did not seem to provide sufficient noise reduction, the image was passed through the filter a second time [7].

\section{DATABASE}

The real medical images like MRI, PET or CT scan and to take up a research is a very complex because of privacy issues and heavy technical hurdles. The purpose of this study is to pre-process brain tumor detection methods through MR brain Images. The MRI data is obtained from the Brain Web Database at the McConnell Brain Imaging centre of the Montreal Neurological Institute (MNI), McGill University [8]. Figure 2 shows sample MRI brain image.
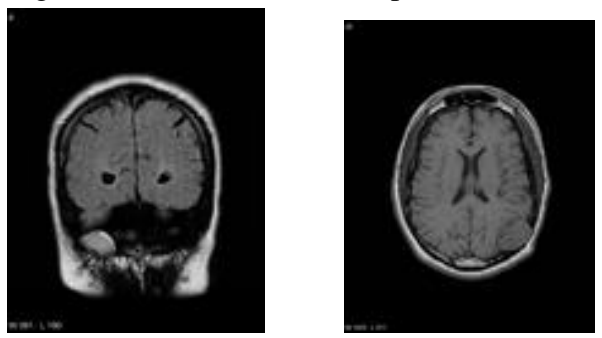

Figure 2 Sample MRI epidermis brain tumor images

Nowadays MRI systems are very important in medical image analysis. Detection of brain tumor requires high resolution brain MRI. Most Medical Imaging Studies and detection are conducted using MRI, Positron Emission Tomography (PET) 
and Computed tomography (CT) Scan. MRI has a multidimensional nature of data provided from different sequential pulses [3]. An MRI scan can evaluate the structure of the heart and aorta, where it can detect aneurysms or tears. MRI scanners can produce 1500 images per second [4].Intraoperative MR imaging can acquire high contrast images of Soft tissue anatomy. MRI is the modality of choice for evaluating brain morphology because it provides superior soft-tissue contrast with flexible data acquisition protocols that highlight several different properties of the tissue [7].

\section{PROPOSED METHOD FOR ENHANCEMENT}

Researchers developed Computer-Aided Diagnosis (CAD) system for Automatic detection of brain tumor through MRI. The CAD system can provide the valuable outlook and accuracy of earlier brain tumor detection. It consists of two stages. First stage has pre-processing and enhancement. Second, feature extraction, feature selection, classification, and performance analysis are compared and studied. Preprocessing and enhancement techniques are used to improve the detection of the suspicious regions in MRI. The enhancement method consists of three processing steps: first, the MRI image is acquired. Second, removal of film artificates such as labels and marks on the MRI image and finally the high frequency components are removed. Segmentation describes separation of suspicious region from background MRI image [9].

Image processing and enhancement stage is the simplest categories of medical image processing. This stage is used for reducing image noise, highlighting edges, or displaying digital images. Some more techniques can employ medical image processing of coherent echo signals prior to image generation. The enhancement stage includes resolution enhancement; contrast enhancement. These are used to suppress noise and imaging of spectral parameters. After this stage the medical image is converted into standard image without noise, film artifacts and labels.

The first step involves the removal of unwanted parts in the background of the MRI Image. The main objective of this process is to improve the quality of the image to make it ready for further processing by removing the irrelevant parts of the image by increasing contrast of the Image using threshold value.

To analyzing MRI image, first segmented the image into very small blocks. To reduce the complexity of the algorithm, degenerate the image into $2 \times 2$ pixel blocks. Checks the intensity or the pixel value of the blocks and calculate the pixel value which present maximum within the block. Propagate the value in the adjacent pixel of the block. Now the entire block contains the same pixel value. So, the whole image now consists of $2 \mathrm{X} 2$ homogeneous blocks.

Now, $2 \mathrm{X} 2$ homogeneous blocks or $4 \mathrm{X} 4$ pixel block, and do the same process as above of $2 \times 2$ block. Now, the whole mammogram image consists of 4X4 homogeneous blocks. Repeat the same process to produce image to the 8 X8 homogeneous blocks.

Noise presented in the image can reduce the capacity of region growing filter to grow large regions or may result as a fault edges. When faced with noisy images, it is usually convenient to pre-process the image by using weighted median filter.

Weighted Median (WM) filters have the robustness and edge preserving capability of the classical median filter. WM filters belong to the broad class of nonlinear filters called stack filters. This enables the use of the tools developed for the latter class in characterizing and analysing the behaviour and properties of WM filters, e.g. noise attenuation capability.

First WM filters are applied on each block for noise attenuation and secondly we applied the following two algorithms for removing the irrelevant parts of the image and increasing contrast of the image using threshold value [10-11].

\section{Algorithm1:}

Input: Pre-processed MRI Image (PMRI)

Isize $=$ Size of the Image

$\mathrm{B}=$ Block Size i.e. 2, 4, 8 etc

$P$ [255] = Calculate the Maximum Occurrence Pixel Value

Iseg [B][1024] = Segment of Image Read Each Time

Output: Homogeneous MRImage (HMRI)

Begin

Step1. Open PMI file.

Step2. Open HI file.

Step3. Loop J=0, Isize/(1024*B)

Read 1024*B from PMRI

Loop I=0, 1024/B

Loop $\mathrm{K}=0,255$

$\mathrm{P}[\mathrm{K}]=0$

$\mathrm{K}=\mathrm{K}+1$

End Loop

Loop $\mathrm{R}=0$, B-1

Loop $\mathrm{C}=\mathrm{I}^{*} \mathrm{~B},\left(\mathrm{I}^{*} \mathrm{~B}\right)+(\mathrm{B}-1)$

$\mathrm{P}[\operatorname{Iseg}[\mathrm{R}][\mathrm{C}]]=\mathrm{P}[\operatorname{Iseg}[\mathrm{R}][\mathrm{C}]]+1$

$\mathrm{C}=\mathrm{C}+1$

End Loop

$\mathrm{R}=\mathrm{R}+1$ 
End Loop

Hvalue $=0$

Maxvalue $=0$

Loop $\mathrm{K}=0,255$

IF Maxvalue <= P $[\mathrm{K}]$

Hvalue $=\mathrm{K}$

Maxvalue $=\mathrm{P}[\mathrm{K}]$

End IF

$\mathrm{K}=\mathrm{K}+1$

End Loop

Loop $\mathrm{R}=0$, B-1

Loop $\mathrm{C}=\mathrm{I}^{*} \mathrm{~B},(\mathrm{I} * \mathrm{~B})+(\mathrm{B}-1)$

Iseg $[\mathrm{R}][\mathrm{C}]=$ Hvalue

$\mathrm{C}=\mathrm{C}+1$

End Loop

$\mathrm{R}=\mathrm{R}+1$

End Loop

$\mathrm{I}=\mathrm{I}+1$

\section{End Loop}

Uniform color quantization technique is used to broken the color space of image into eight equal sized regions. Although color quantization is a lossy process but we utilized this due to sampling the original image for color statistics, choosing a color map based on those statistics and mapping the colors to their representative properties in the color map. The $8 \mathrm{X} 8$ homogeneous image is now clearly segmented into different color region and each region representing specific parts and properties.

\section{Algorithm2:}

Input: Homogeneous Image (HI)

Isize $=$ Size of the Image

Byte $=$ one byte of data offset

Pbyte $=$ to hold the previous value of byte

Output: Color Quantized Image (CQI)

Begin

Step1. Open PMI file

Step2. Open HI file

Step3. Loop J=0, Isize

Read one Byte from HI Loop I=0, 256

IF Byte < I

Byte = I - 32

Break

End IF

$\mathrm{I}=\mathrm{I}+32$

End Loop

IF J=0

Pbyte $=$ Byte

ELSE

IF Pbyte-Byte>32 OR

Byte-Pbyte $>32$

ELSE

Pbyte $=$ Byte

End IF Byte $=$ Pbyte

End IF

Write one byte to CQI

End Loop $\mathrm{J}=\mathrm{J}+1$

End Step4. Close HI, CQI

Figure below shows the results.

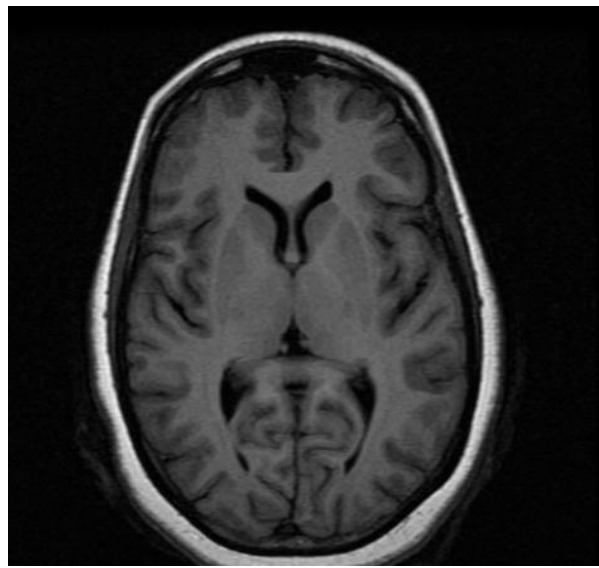

Original Image 2 


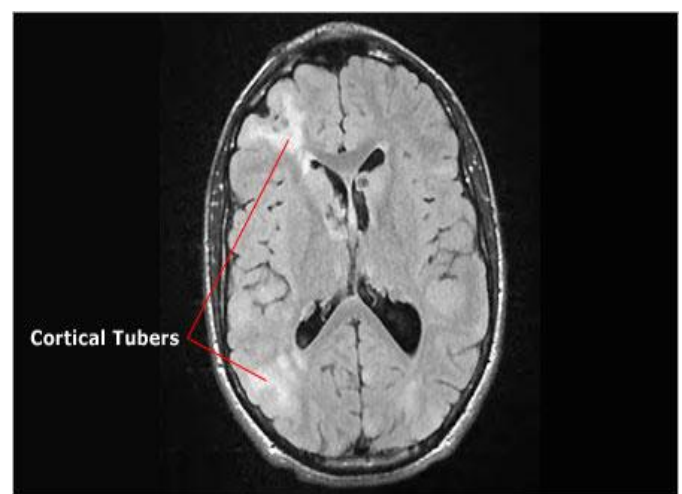

After Pre-processing of Image 1

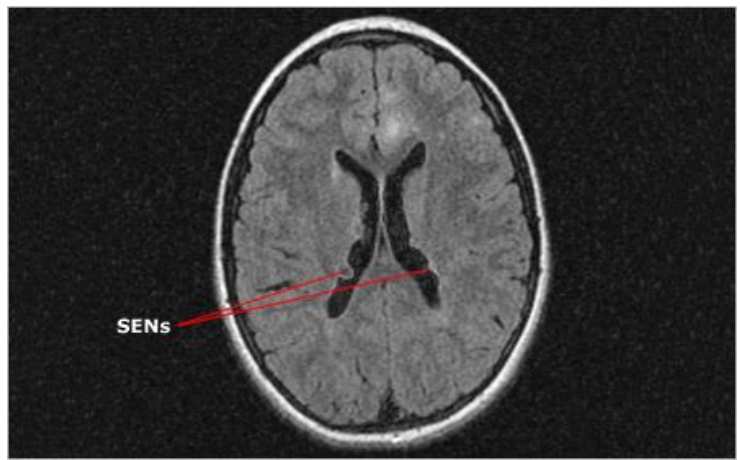

After Pre-processing of Image 1
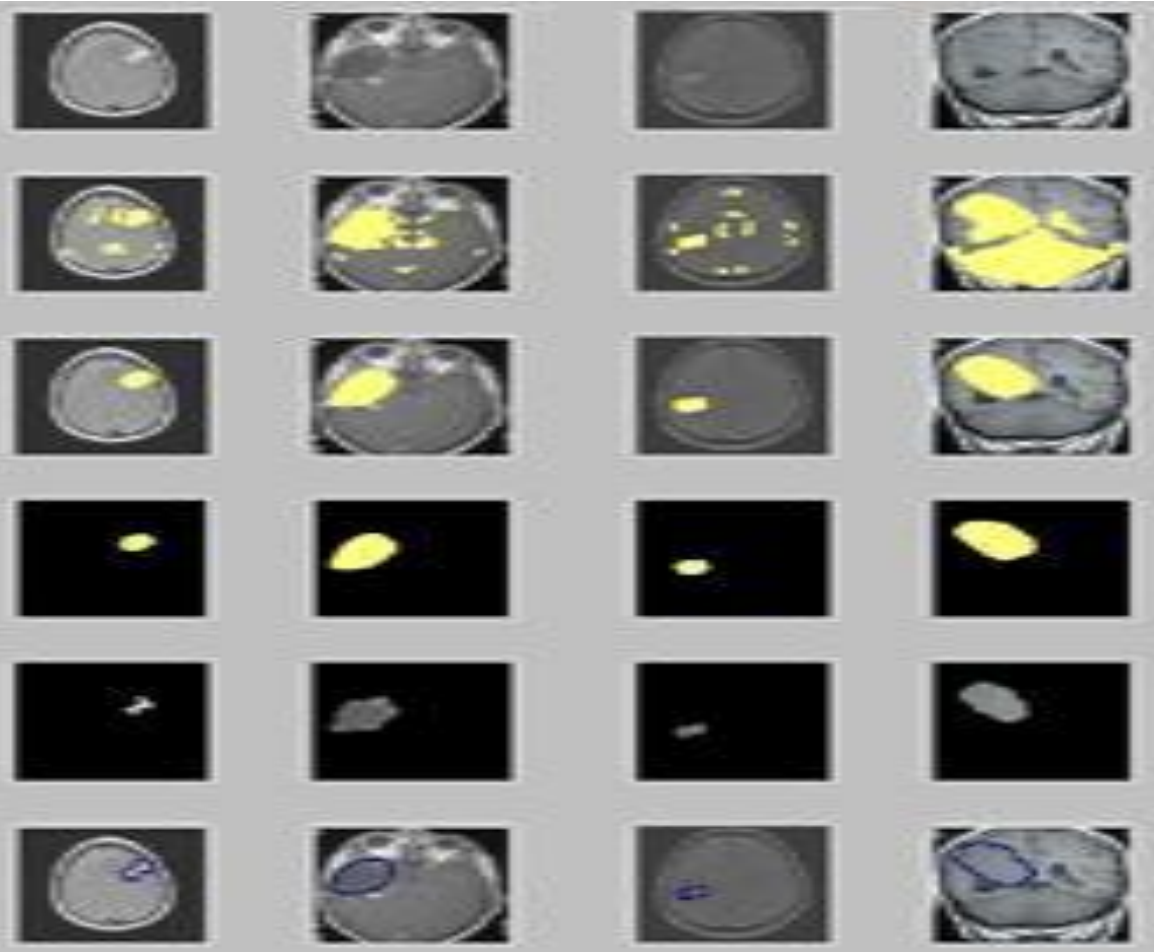

Starting from Original Images (row 1), Pre-processed Image (row 2 and 3), Images Applying Algorithm 1 (row 3), Isolation (row 4), Gray Image (row 5), and Output Image (row 6)

\section{CONCLUSION}

Low-field brain MRI is now being introduced in medical institutions for real-time imaging during surgeries. However, it produces low-resolution images and leads to difficulty in automated segmentation techniques. we managed to eliminate the unnecessary background and artifacts in the image. The clarity of the ROI was enhanced effectively while almost no loss of information or image 
integrity was recorded in the actual brain areas of the processed low field MRI images.

\section{REFERENCES}

[1] U. Vovk, F. Pernu, B. Likar, "Simultaneous correlation of intensity inhomogeneity in multichannel MR images", 27th Annual Conf. Engineering in Medicine and Biology, pp. 4290-4293, 2005.

[2] N.I. Weisenfeld, S.K. Warfield, "Normalization of joint image-intensity statistics in MRI using the KullbackLeibler divergence", IEEE Symposium on Biomedical Imaging : Nano to Macro, vol. 1, pp. 101-104, 2004.

[3] A. Madabhushi, J.K. Udupa, "Interplay between intensity standardization and inhomogeneity correction in MR image processing", IEEE Trans. on Medical Imaging, vol. 24, pp. 561-576, 2005.

[4] U. Vovk, F. Pernus, B. Likar, "A review of methods for correction of intensity inhomogeneity in MRI", IEEE Trans. on Medical Imaging, vol. 26, pp. 405-421, 2007.

[5] Y.L. Liao, N.T. Chiu, C.M. Weng, Y.N. Sun, "Registration and normalization techniques for assessing brain functional images", Biomedical Engineering Applications, Basis \& Communications, 2003.
[6] J.G. Park, T. Jeong, C. Lee, "Automated brain segmentation algorithm for 3D magnetic resonance brain images", 2nd Intl. Workshop on Soft Computing Applications (SOFA), pp. 57- 61, 2007.

[7] Majos C, Julia-Sape M, Alonso J, Serrallonga M, Aguilera C, Juan J, Gilli J. Brain tumor classification by proton MR spectroscopy: Comparison of diagnostic accuracy at short and long TE. AJNR 2004;25:1696-704.

[8] Li G, Yang J, Ye C, Geng D. Degree prediction of malignancy in brain glioma using support vector machines. Computers in Biology and Medicine 2006;36:313-25.

[9] Messen W, Wehrens R, Buydens L. Supervised Kohonen networks for classification problems. Chemometrics and Intelligent Laboratory Systems 2006; 83:99-113.

[10] Chaplot S, Patnaik M, Jagannathan N. Classification of magnetic resonance brain images using wavelets as input to support vector machine and neural network. Biomedical Signal Processing and Control 2006;1:86-92.

[11] Luts J, Heerschap A, Johan A, Huffel S. A combined MRI and MRSI based multiclass system for brain tumour recognition using LS-SVMs with class probabilities and feature selection. Artificial Intelligence in Medicine 2007;40:87-102. 